International Journal of Modern Physics A, (C) World Scientific Publishing Company

\title{
PRODUCT INTEGRALS AND WILSON LOOPS
}

\author{
ROBERT L. KARP * \\ Physics Department, University of Cincinnati \\ Cincinnati, Ohio 45221-0011, USA
}

\begin{abstract}
Using product integrals we review the unambiguous mathematical representation of Wilson line and Wilson loop operators, including their behavior under gauge transformations and the non-abelian Stokes theorem. Interesting consistency conditions among Wilson lines are also presented.
\end{abstract}

Wilson loops provide a convenient method of obtaining gauge invariant observables from lattices to strings. On the other hand the product integral formalism was developed in connection with matrix valued differential equations, having built in the feature of order of the matrices. Consequently these come quite handy when dealing with path ordered quantities. As the theory of product integrals is well founded, we can use the various results in the subject to study Wilson lines and loops. In this paper we review the mathematics of the formalism of product integrals and its applications to the physics of Wilson lines and Wilson loops.

\section{Review of product integration}

The long history of product integration starts with Volterral. In fact the product integral is to the product what the ordinary integral is to the sum. For starting point consider an evolution equation of the type $\mathbf{Y}^{\prime}(\mathbf{s})=A(s) \mathbf{Y}(\mathbf{x})$ on the interval $[a, b]$. Given $\mathbf{Y}(a)$ we want to find $\mathbf{Y}(b)$. We can obtain an approximate value

$$
\mathbf{Y}(b) \approx e^{A\left(s_{n}\right)\left(s_{n}-s_{n-1}\right)} \ldots e^{A\left(s_{1}\right)\left(s_{1}-s_{0}\right)} \mathbf{Y}(a) \equiv \Pi_{P}(A) \mathbf{Y}(a)
$$

for any partition $P=\left\{s_{0}, s_{1}, \ldots, s_{n}\right\}$ of the interval $[a, b]$. In fact this approximation process can be made completely precise, and in the limit where the norm of the partition $\mu(P)$ goes to zero, under suitable conditions, the expression (1) converges to the product integral: $\mathbf{Y}(b)=\lim _{\mu(P) \rightarrow 0} \Pi_{P}(A) \mathbf{Y}(a)$.

We briefly enumerate some of the basic properties of product integrals明. First we note that $\prod_{a}^{x} e^{A(s) d s}$ is the solution of the initial value problem we started with. From the formula $\operatorname{det}\left(\prod_{a}^{x} e^{A(s) d s}\right)=e^{\int_{a}^{x} \operatorname{Tr} A(s) d s}$ we infer that product integrals are non-singular matrices. We have a composition rule similar to that of ordinary integrals: $\prod_{a}^{b} e^{A(s) d s}=\prod_{c}^{b} e^{A(s) d s} \prod_{a}^{c} e^{A(s) d s}$. The rule of differentia-

\footnotetext{
*Present address: Department of Mathematics, Duke University, Durham, NC 27708.
} 
tion with respect to the endpoints reads: $\frac{\partial}{\partial x}\left(\prod_{y}^{x} e^{A(s) d s}\right)=A(x) \prod_{y}^{x} e^{A(s) d s}$ and $\frac{\partial}{\partial y}\left(\prod_{y}^{x} e^{A(s) d s}\right)=-\prod_{y}^{x} e^{A(s) d s} A(y)$.

The usual way of computing ordinary integrals is by the means of the NewtonLeibnitz formula, involving primitive functions. The analogous notion for product integrals uses the so-called L-operation. The L-derivative for a nonsingular matrix valued function $P:[a, b] \rightarrow \mathbf{C}_{n \times n}$ is defined by: $L P(x):=P^{\prime}(x) P^{-1}(x)$. It is immediate that $\prod_{a}^{b} e^{(L P)(s) d s}=P(b) P^{-1}(a)$, the analog of the well known NewtonLeibnitz formula.

We reproduce three more important theorems that will be of great use later on: Sum rule: $\prod_{a}^{x} e^{[A(s)+B(s)] d s}=P(x) \prod_{a}^{x} e^{P^{-1}(s) B(s) P(s) d s}$, where $P(x)=\prod_{a}^{x} e^{A(s) d s}$. Similarity rule: $P(x)\left(\prod_{a}^{x} e^{B(s) d s}\right) P^{-1}(a)=\prod_{a}^{x} e^{\left[L P(s)+P(s) B(s) P^{-1}(s)\right] d s}$.

Derivative with respect to a parameter: For $A(s ; \lambda)$ with the right properties 1 we have: $\frac{\partial}{\partial \lambda} \prod_{y}^{x} e^{A(s ; \lambda) d s}=\int_{y}^{x} d s \prod_{s}^{x} e^{A(s ; \lambda) d s} \frac{\partial A}{\partial \lambda}(s ; \lambda) \prod_{y}^{s} e^{A(s ; \lambda) d s}$.

\section{Wilson lines and Wilson loops}

Let $M$ be an n-dimensional manifold representing the space-time and $A$ a Liealgebra valued connection one-form $A(x)=A_{\mu}^{k}(x) T_{k} d x^{\mu}$. For a curve $C$ (closed) the Wilson line (loop) is defined as $W[C]=\mathcal{P} e^{\int_{a}^{b} A}$. If $C$ is parametrized by $\sigma$ then $A(x)=A(\sigma) d \sigma$, where $A(\sigma) \equiv A^{\mu}(x(\sigma)) d x^{\mu} / d \sigma . A(\sigma)$ plays the role of the matrix function in the product integral.

Let us next consider the Wilson loop. For simplicity we assume that the loop may be taken to be the boundary of a two dimensional orientable surface $\Sigma$ parametrized by $(\sigma, \tau)$. We can construct the pulled-back field strength $F_{a b}$ in two different ways: as the the field strength of the pulled-back connection or as the pull-back of the field strength. It is easy to check that the two coincide.

Our goal is to express the Wilson loop operator in terms of product integrals. As the Wilson loop depends only on the homotopy class of $C$, we can parameterize $C$ conveniently: break it up into segments along which either $\sigma$ or $\tau$ remains constant. The composition rule for product integrals ensures that the final result is independent of the intermediate points. Accordingly we have $W[C]=W_{4} W_{3} W_{2} W_{1}$. For these intermediate Wilson lines $\sigma_{0}=$ const. along $W_{1}$ and $W_{3}$, and $\sigma_{1}=$ const. along $W_{2}$ and $W_{4}$.

Based on the properties of product integrals, it was shown by two independent methods ${ }^{2}$ that $W[C]$ has a surface integral representation of the form:

$$
W=\prod_{\tau_{0}}^{\tau} e^{\int_{\sigma_{0}}^{\sigma} T^{-1}\left(\sigma^{\prime} ; \tau^{\prime}\right) F_{01}\left(\sigma^{\prime} ; \tau^{\prime}\right) T\left(\sigma^{\prime} ; \tau^{\prime}\right) d \sigma^{\prime} d \tau^{\prime}}
$$

where $T$ is a given Wilson line. This can be interpreted as a non-abelian Stokes theorem.

Let us now focus on the transformation properties of Wilson lines and Wilson loops under a gauge transformation $A(x) \longrightarrow g(x) A(x) g^{-1}(x)-g(x) d g(x)^{-1}$. It 
was shownl using product integration that Wilson lines have the expected transformation $\mathcal{P} e^{\int_{a}^{b} A} \longrightarrow g(b)\left(\mathcal{P} e^{\int_{a}^{b} A}\right) g(a)$. For the Wilson loop one has two ways to derive the transformation property: use the already established one for the lines, or use the surface integral representation provided by the non-abelian Stokes theorem (2). It was checked 1 that both ways we get the well established answer, reinforcing our confidence in the techniques used.

\section{Further properties}

It is interesting to use the product integral formalism to derive interesting identities among Wilson lines. For brevity we introduce the following notation: $P(A ; b, a)=\prod_{a}^{b} e^{A(s) d s}$. Observing that $\prod_{a}^{b} e^{A(s) d s}=\prod_{a}^{b} e^{[\lambda A(s)+(1-\lambda) A(s)] d s}$ for any real $\lambda$, based on the sum rule we can deduce immediately that

$$
P(\lambda A ; a, b) P(A ; b, a)=\prod_{a}^{b} e^{P(\lambda A ; a, s)(1-\lambda) A(s) P(\lambda A ; s, a) d s} .
$$

An interesting immediate application of (3) is the addition law we obtain for $\lambda=\frac{1}{2}$ : $P(2 A ; b, a)=P(A ; b, a) \prod_{a}^{b} e^{P(A ; a, s) A(s) P(A ; s, a) d s}$.

Another application of (3) is by differentiating with respect to $\lambda$. After some algebra, involving the formula for differentiation with respect to a parameter recalled earlier, we obtain the following result:

$$
\begin{gathered}
\int_{a}^{b} d s\{P(\lambda A ; a, s) A(s) P(\lambda A ; s, b) P(A ; b, a)-P(\lambda A ; s, b) P(A ; b, s) \\
{\left[-P(\lambda A ; a, s) A(s)+(1-\lambda) \int_{a}^{s} d t[P(\lambda A ; a, s) A(s) P(\lambda A ; s, t) A(t) P(\lambda A ; t, s)\right.} \\
+P(\lambda A ; a, t) A(t) P(\lambda A ; t, s) A(s)] P(A ; s, a)]\}=0 .
\end{gathered}
$$

In conclusion we note that the results described here were extended to the super-

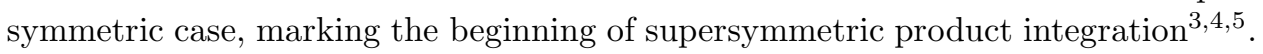

\section{Acknowledgements}

Most of the results presented here were obtained in an enjoyable collaboration with F. Mansouri. This work was supported in part by the Department of Energy under the contract number DOE-FGO2-84ER40153.

\section{References}

1. J.D. Dollard, C.N. Friedman, Product Integration, Addison Wesley, 1979.

2. R. L. Karp, F. Mansouri and J. S. Rno, Turk. J. Phys. 24, 365 (2000) hep-th/9903221]; J. Math. Phys. 40, 6033 (1999) hep-th/9910173.

3. R. L. Karp and F. Mansouri, Phys. Lett. B480, 213 (2000) hep-th/0002085.

4. R. L. Karp, F. Mansouri, To appear in the Proceedings of the Symposium on Quantum Theory and Symmetry, 1999 Goslar, Germany.

5. R. L. Karp, F. Mansouri, To appear in the Proceedings of the Orbis Scientiae '98, Ft. Lauderdale, FL. 